\title{
Servicio de transporte urbano en la ciudad de Lima, análisis y propuesta de mejora de la calidad, con participación de las universidades públicas y privadas
}

\author{
Public transportation service in Lima: analysis and proposal to improve its quality, with participation \\ of public and private universities
}

\author{
Josué G. Centeno C. ${ }^{1}$
}

\begin{abstract}
Resumen
El servicio de transporte urbano en la ciudad de Lima es de baja calidad y tiende a empeorar debido a la falta de atención de parte del Sector Público, del incremento progresivo de los usuarios y la falta de interés por parte de los ofertantes del servicio por mejorar su oferta. Los estudiantes universitarios por disposición del gobierno gozan de una tarifa preferencial denominada "pasaje universitario" que es el 50\% de un pasaje de adulto que deben de otorgar tanto los sistemas de transporte de propiedad del estado como las empresas privadas. Uno de los principales factores de la mala calidad es la falta de capacitación de los propietarios, chóferes y cobradores para dar una buena atención que satisfaga a todos los usuarios. Los beneficiarios del pasaje universitario pueden contribuir a manera de retribución por el beneficio recibido, participando activamente en mejorar la calidad del servicio creando en la sociedad una cultura de responsabilidad. El presente artículo plantea la posibilidad de articular un trabajo conjunto entre los beneficiarios del pasaje universitario, los operadores de los servicios de transporte urbano, los propietarios de las unidades de transporte y los órganos del gobierno a través de programas de capacitación diseñados y conducidos por las universidades, para contribuir a mejorar la calidad del sistema de transporte en la ciudad de Lima.
\end{abstract}

Palabras clave: transporte urbano; pasaje universitario; calidad; operadores; capacitación.

\begin{abstract}
Public Transportation in Lima has a low quality standard and tend to get worse due to lack of attention of the Public Sector, to the permanent increase of users and lack of interest of suppliers to offer a better service. University students by law have a lower rate called "university ticket" which is equivalent to $50 \%$ of a normal adult fare. Public transportation companies as well as private companies are obliged to charge this lower rate. One of the factors that contribute to low quality service is the lack of training of owners, drivers and collectors to give high standard service that could satisfy client expectations. The beneficiaries of the "university ticket" could repay the benefit by actively participating in improving the quality of the service creating in society a culture of responsibility. This article proposes a joint work between the beneficiaries of the "university ticket", the operators of the public transportation, the owners of the transportation units and the government institutions to improve the quality of the transportation system in Lima through capacitation programs designed and conducted by universities.
\end{abstract}

Keywords: public Transportation; university ticket; quality; operators; capacitation.

\section{Introducción}

El sistema del servicio de transporte urbano, descansa en tres subsistemas. El Subsistema Normativo o de regulación, el Subsistema de capacitación y el Subsistema de infraestructura. El Subsistema normativo o de regulación es el que se encarga de dar las normas, leyes, reglamentos, disposiciones de cumplimiento obligatorio relacionados al otorgamiento de licencias, concesión de rutas, números de unidades por rutas, regulación referente a la constitución de empresas etc. Lo integran las entidades del Estado como el Ministerio de Transportes, Municipalidades, Ministerio del Interior etc. (Sector Público). El Subsistema de capacitación, son todas aquellas actividades relacionadas al mejor cumplimiento de las normas y a la enseñanza de los actores vinculados a este servicio a fin de brindar satisfacción a los usuarios. (Sector Público y Sector Privado) y el Subsistema de Infraestructura relacionada a proporcionar los elementos físicos tales como vías de circulación (pistas y carreteras), señalizaciones (avisos, semáforos) vehículos, (ómnibuses, micros, combis) para cumplir eficientemente este servicio.

La población estudiantil universitaria como parte de los usuarios, hace uso del servicio de transporte urbano en condiciones especiales relacionadas a la concesión de una tarifa diferenciada denominada "pasaje universitario", que es el $50 \%$ del pasaje adulto. 
El manejo, monitoreo y diseño de los subsistemas normativos e infraestructura son de responsabilidad de las autoridades del gobierno central y municipal al otorgar las licencias y concesiones de rutas y clasificación de las unidades de transporte, de las empresas privadas y/o públicas, al otorgar las concesiones de rutas, clasificación de unidades de transporte licencias etc. y el subsistema de infraestructura en lo relacionado a las unidades de transporte que brindan el servicio, son de responsabilidad de las empresas privadas y públicas. El presente trabajo solo enfocará el subsistema relacionado a la capacitación de los que proveen directamente el servicio y que tienen contacto directo con los usuarios.

El presente artículo tiene el objetivo investigar la percepción y los niveles de satisfacción que tienen los estudiantes universitarios sobre la calidad del transporte urbano en la ciudad de Lima, determinar el gasto anual que representó el transporte urbano para los estudiantes universitarios y proponer la participación del sistema universitario a través de sus estudiantes en la mejora de la calidad del servicio de transporte urbano como una contraparte del privilegio que se tiene al pagar el 50\% como pasaje concedido a los estudiantes universitarios.

\section{Materiales y métodos}

El presente trabajo se realizó en la ciudad de Lima, en el área geografía de Lima Metropolitana, donde se encuentran los estudiantes universitarios que gozan del pasaje universitario, y donde operan las unidades de transporte de las empresas públicas y privadas.

La presente investigación corresponde al método de tipo exploratorio, considerando que existe muy poca información que relacione la satisfacción que tienen los usuarios sobre los servicios recibidos en el sistema de transporte urbano y programas o proyectos que contribuyan a mejorar la calidad de los servicios.

La muestra que se ha tomado como materia de investigación ha sido los estudiantes matriculados en las diferentes universidades de Lima Metropolitana y que de acuerdo a los carnets emitidos por la Asamblea Nacional de Rectores (ANR), tiene derecho al pasaje universitario. Solo se considera a los que están en este sistema más no a los escolares ni estudiantes de institutos superiores que también tienen acceso a este beneficio. La información obtenida de las municipalidades y de los órganos del gobierno central, son las que se han utilizado para este fin. No existe mayor información de parte de los empresarios y propietarios de las unidades de transporte puesto que muchos de ellos son informales y tiene cierta resistencia a dar información por diferentes temores que les acompaña.

\section{Técnicas de recolección de datos}

Documentación y análisis: Se utilizó artículos y documentos de carácter oficial en algunos casos y en otros se acudió a la información disponible en la web de los actores $\mathrm{y}$ en otros se tuvo que elaborar la información recogiendo de observaciones en ciertos puntos de concurrencia masiva de los usuarios. Se tuvo acceso a redes y blocks de los actores del sistema.

Encuestas y entrevistas: Se realizaron encuestas a los estudiantes de universidades públicas y privadas para determinar su satisfacción por el servicio así como el gasto que tienen que incurrir en los pasajes urbanos, así mismo se encuestó a diferentes usuarios del sistema de transporte ya sea para investigar su nivel de satisfacción o aquellos aspectos en los cuales perciben que debe de mejorar.

\section{Procedimiento de análisis de datos}

El procedimiento utilizado para el análisis de los datos obtenidos se realizó de la siguiente manera: a) Recolección de la información mediante el diseño de las encuestas preparadas, b) Procesamiento asignando en unos casos códigos o indicadores c) Articulación de la información obtenida d) Interpretación de los resultados.

\section{Limitaciones}

El sistema de transporte en Lima no está articulado, existe mucha informalidad y como consecuencia la información que se ha obtenido es solo aquella que se considera confiable o que fueron publicados por los medios oficiales, sin embargo se han hecho algunos reajustes en base a la información que se pudo obtener de las propias fuentes.

\section{Resultados y discusión}

\section{Oferta de unidades al sistema de transporte}

El transporte urbano de Lima es ofertado por las unidades de transporte de propiedad del estado (Municipalidades tales como trenes, ómnibus etc.) y las empresas privadas para cuyo efecto deben de previamente realizar trámites ante las autoridades municipales y del gobierno central para obtener las respectivas licencias de funcionamiento.

Para el presente caso solamente nos referiremos a la oferta de las empresas privadas que están formalizadas y realizan el servicio de transporte de una manera regular.

La clasificación a las diferentes unidades está determinada por la capacidad de transporte siendo la siguiente la adoptada por la Municipalidad de Lima y el Ministerio de Transporte: Ómnibus: Vehículo con capacidad de más de 40 pasajeros. Microbús: Vehículo con capacidad de 21-40 pasajeros. Camioneta Rural (Combi): Vehículo con capacidad de 12 Pasajeros. La antigüedad promedio por vehículo es de 20 años. El tamaño de flota es, en promedio, de 60 unidades, en las camionetas rurales (combis) por ruta, siendo las rutas de ómnibus y microbuses las de mayor tamaño de flota, con tamaños promedio de 68 y 61 vehículos por ruta, respectivamente. Estas unidades están agrupadas en 380 empresas.

La Tabla 1 muestra que la oferta está constituida por un total de 23182 unidades, entre camionetas rurales (combis), microbuses y ómnibus. En particular, se observa que en Lima predominan unidades pequeñas y antiguas: 
Tabla 1. Unidades autorizadas por la municipalidad para el servicio las universidades se ha producido a partir del año de transporte urbano Lima (2013)

\begin{tabular}{lccc}
\hline & Ómnibus & Microbús & Camioneta Rural \\
\hline Cantidad & 2783 & 11359 & 9040 \\
Ingreso Prom. diario S/. & 313 & 243 & 183 \\
Ganancia mensual S/. & 3,091 & 3,117 & 2221 \\
\hline Apoyo Consultores
\end{tabular}
2010. De igual modo la población universitaria se ha incrementado en los últimos años tal como se señala a continuación.

La Tabla 3 muestra que la población universitaria procedentes de universidades públicas, en Lima se ha incrementado en los últimos años pasando de

Apoyo Consultores

Según reportes de la Gerencia de Transporte Urbano de la Municipalidad Metropolitana de Lima (2013), el 49\% de las unidades son microbuses; el 39\%, camionetas rurales y solo el $12 \%$, ómnibus. La misma que se muestra en la Tabla 1.

Además se muestra que el ingreso promedio diario tiende a ser mayor para los vehículos de mayor tamaño, los cuales pueden acoger a un mayor número de pasajeros. Así, el propietario de un ómnibus percibe $28 \%$ más ingresos que el propietario de un microbús y $70 \%$ más que el propietario de una camioneta rural. Por su parte, el propietario de un microbús recibe $32 \%$ más ingresos que el propietario de una camioneta rural. Sin embargo, pese a generar mayores ingresos, los vehículos de mayor tamaño también generan mayores gastos, especialmente en lo concerniente a mantenimiento. Estos mayores gastos reducen el margen de ganancia de los propietarios. A diferencia de los costos de cochera o combustible, los costos de mantenimiento responden a la antigüedad por lo que su impacto es mayor conforme la antigüedad del vehículo.

Con respecto a las ganancias de propietarios, se observa que las ganancias absolutas tienden a ser mayores para los propietarios de ómnibus y microbuses que para los propietarios de camionetas rurales. Así, por ejemplo, la ganancia mensual absoluta de los propietarios de ómnibus es un $25 \%$ mayor a las ganancias de los propietarios de las 97,317 estudiantes en el año 2008 a 107,527 en el año 2013 , es decir un crecimiento de $10.5 \%$.

Los alumnos matriculados en universidades privadas en el año 2008 eran de 227,735 y en el año 2013 incrementó a 268,450, es decir tuvo un aumentó de 17.9 $\%$, es decir creció en mayor número que los estudiantes de universidades púbicas.

\section{La calidad en el sistema de transporte urbano}

Uno de los servicios más utilizados y que más quejas e insatisfacciones producen, es el sistema de transporte urbano. Las disposiciones que se han dado para solucionar la creciente demanda de unidades, ha dado como resultado que se produzca una proliferación de empresas y unidades de transporte y juntamente con ello el incremento de operadores (chóferes cobradores) quienes no se preocupan de mejorar la calidad del servicio sino por el contrario la de ponerse en serias competencias agresivas entre ellos para poder sacar la mayor utilidad posible en el más corto plazo. Producto de ello, se ha creado una cultura "combi" como una designación de procedimientos informales, de pésima calidad y de permanentes muestras de insatisfacción por parte de los usuarios. Si bien es cierto, es un secreto a voces este descontento, muy poco se hace para mejorar los servicios, echándose la culpa entre los propietarios de las unidades de vehículos, el gobierno municipal o el gobierno central. camionetas rurales, tal como se aprecia en la Tabla 1. Se observa también que las mayores ganancias las obtienen los propietarios de microbuses, aunque esta no es significativamente mayor que la ganancia de los propietarios de ómnibus. En vista que la diferencia entre la ganancia de los propietarios de ómnibus y microbús no es significativa, se puede afirmar que los propietarios de ómnibus y microbús obtienen ganancias similares en el mercado.

Tabla 2. Ubicación geográfica de las universidades en el país (año 2013)

\begin{tabular}{lccc}
\hline Ubicación geográfica & Pública & Privada & Total \\
\hline Consejo Regional Interuniversitario Norte & 11 & 15 & 26 \\
Consejo Regional Interuniversitario Lima & 12 & 43 & 55 \\
Consejo Regional Interuniversitario Amazónico & 9 & 5 & 14 \\
Consejo Regional Interuniversitario Centro & 8 & 9 & 17 \\
Consejo Regional Interuniversitario Sur & 11 & 17 & 28 \\
\hline Total & $\mathbf{5 1}$ & $\mathbf{8 9}$ & $\mathbf{1 4 0}$ \\
\hline
\end{tabular}

Tabla 3. Crecimiento poblacional de estudiantes universitarios de pregrado (Lima)

\section{Demanda universitaria de transporte}

La demanda del transporte urbano para los estudiantes universitarios de la capital proviene de las universidades existentes en Lima la misma que se muestra en la Tabla 2. Esto indica que la mayor cantidad de universidades se encuentran en la región de Lima constituyendo 12 públicas y 43 privadas haciendo un total de 55 universidades. El crecimiento cuantitativo de

\begin{tabular}{lccc}
\hline Año & Universidad Pública & Universidad Privada & Total \\
\hline 2008 & 97317 & 227735 & 325052 \\
2010 & 104294 & 245908 & 351912 \\
2013 & 107527 & 268450 & 377990 \\
\hline
\end{tabular}

Fuente : INEI (2016)

\section{Insatisfacción de los usuarios (público en general)}

Es necesario encontrar cuales son las razones principales de su insatisfacción, para realizar la calificación de acuerdo a la percepción. 
Se ha tomado como punto de partida para esta evaluación, las quejas proveniente de diferentes sectores sociales que ha recibido la defensoría del pueblo en el año 2008, donde se registraton los motivos de las quejas, así como el número de ellas (Total 136).

Las mayores quejas del público, viene por la indisciplina en la ruta y los horarios que no cumplen los chóferes y cobradores (38.23\%) seguido por el maltrato que reciben por parte de los operadores $(26.47 \%)$ luego la alteración de precios de los pasajes $(18.39 \%)$ y finalmente la temeridad y falta de comodidad e higiene $10.3 \%$ y $6.62 \%$ respectivamente (Tabla 4 ).

Para poder medir el nivel de insatisfacción del usuario, era necesario establecer una tabla que fuera capaz de cuantificar los diferentes niveles de insatisfacción para de esa manera encontrar cuales eran aquellos motivos o circunstancias que el usuario considera que le produce mayor insatisfacción o ninguna insatisfacción. Esta tabla a su vez ayuda a priorizar la solución de aquellas que se considera las causas que producen las mayores insatisfacciones así poder priorizar las propuestas para una solución efectiva. considera en términos generales.

Siendo el universo de estudiantes universitarios quienes son el motivo de la presente investigación se ha realizado encuestas entre los estudiantes de las universidades públicas y privadas para poder obtener una información objetiva de su percepción sobre el sistema de transporte urbano que recibe puesto que ellos son los que más frecuentemente utilizan.

Pésimo (1) Considera que el servicio deja mucho que desear, el usuario sale descontento con deseos de no volver a utilizar. Solo se utiliza porque no hay otro medio.

Muy malo (2) Considera que el servicio no cumple con las reglas de seguridad, no hay regularidad, no percibe una solución a corto plazo.

Regular (3) Condiciones del servicio medianamente aceptables. Indica que por el precio que se paga no se puede exigir más. El usuario se acomoda al servicio y espera que en algún momento pueda mejorar.
Tabla 4. Quejas de los usuarios relacionados al servicio de transporte (año 2008)

\begin{tabular}{|c|c|c|}
\hline Tipo de queja & No. De quejas & $\%$ \\
\hline \multicolumn{3}{|l|}{ Incomodidad e higiene del vehículo (6.62\%) } \\
\hline Exceso de pasajeros e higiene & 2 & 1.47 \\
\hline Tocar con exceso el claxon & 2 & 1.47 \\
\hline No respetar el asiento reservado & 2 & 1.47 \\
\hline Asientos incómodos & 2 & 1.47 \\
\hline Alto volumen de la radio & 1 & 0.74 \\
\hline \multicolumn{3}{|l|}{ Trato del cobrador y/o chofer $(26.47 \%)$} \\
\hline Agresión física o verbal & 36 & 26.47 \\
\hline \multicolumn{3}{|l|}{ Alteración de precios (18.39\%) } \\
\hline No entregar vuelto & 1 & 0.74 \\
\hline Cobrar tarifa distinta & 24 & 17.65 \\
\hline \multicolumn{3}{|c|}{ Temeridad e informalidad durante el recorrido $(10.3 \%)$} \\
\hline Velocidad no razonable & 9 & 6.62 \\
\hline $\begin{array}{l}\text { Maniobra imprudente no recoger pasajeros, } \\
\text { paradas en lugares no autorizados }\end{array}$ & 5 & 3.68 \\
\hline \multicolumn{3}{|l|}{ Indisciplina en la ruta y horarios $(38.23 \%)$} \\
\hline No cumplir recorrido de ruta & 32 & 23.53 \\
\hline No recoger pasajeros & 12 & 8.82 \\
\hline Vehículo no registrado (pirata) & 8 & 5.88 \\
\hline Total & 136 & \\
\hline
\end{tabular}

\section{Percepción de la calidad del transporte urbano}

No existen parámetros oficiales para medir y clasificar la percepción que tienen los usuarios de la calidad del servicio de transporte en general. Para tener un parámetro comparativo, se ha elaborado una clasificación que permite medir la percepción de la calidad del transporte urbano lo que nos permitirá tener referentes cualitativos para medir la percepción de la calidad del servicio de transporte urbano considerando la infraestructura, reglamentación y todo aquello de carácter interno y externo que el usuario
Bueno (4) Servicio aceptable, hay regularidad en los tiempos y la comodidad brindada permite un viaje adecuado.

Muy bueno (5) Servicio excelente, hay orden y limpieza, buen trato y da gusto viajar.

\section{Niveles de satisfacción/insatisfacción de los usuarios}

Los usuarios manifiestan diferentes niveles de insatisfacción cuando hacen uso los servicios del transporte urbano. Tampoco existen indicadores oficiales que permitan cuantificar el nivel de insatisfacción que produce el uso de los servicios de transporte público (Figura 1). Esta medición considera el grado de satisfacción que sienten cuando son tratados por los que otorgan el servicio al interior de la unidad transportadora.

Mucha Insatisfacción. El usuario se siente muy incómodo por la falta de comodidades. No llega a su destino oportunamente. Hay falta de higiene. Le causa mucha fatiga y estrés el uso de este sistema de transporte.

Insatisfacción. El usuario tiene insatisfacción pero desea que al más breve plazo esta situación mejore. No tolera algunas manifestaciones del mal servicio. Puede soportar ciertas incomodidades. 
Indiferente. Prefiere ignorar el tipo de servicio. Se resigna con tal de llegar a su destino. Prefiere hacer algo (leer, escuchar música) a fin no percibir las causas de incomodidad.

Poca Insatisfacción. Se resigna y admite que estamos en el Perú, y que alguna vez cambiará No hace nada por cambiar, le interesa solamente pasarla hasta llegar a su destino.

Ninguna Insatisfacción. Se siente cómodo, el viaje le puede resultar hasta placentero porque tiene motivos para a distraerse y aprovechar de alguna manera el tiempo del viaje.

Del análisis de la Figura 1 se puede deducir que las mayores insatisfacciones para los estudiantes de las universidades el maltrato del cobrador y chófer $(26 \%)$ seguido de la falta de comodidad e higiene (22.4\%) luego viene la indisciplina de los chóferes y cobradores (22.1\%) seguido muy de cerca de la temeridad en la conducción de las unidades vehiculares. No es de mucha insatisfacción la alteración de precios lo que indica que estarían dispuestos a soportar estos hechos con tal de tener un servicio adecuado

\section{Gastos en pasajes que realizan los estudiantes universitarios}

Los gastos en pasajes que hacen los estudiantes de universidades públicas y privadas son diferentes. Para determinar cuánto gasta un estudiante universitario en pasajes, se procedió a realizar encuestas en forma directa para determinar cuál era la frecuencia diaria o semanal que usaban los servicios de transporte público y cuál era el gasto en los cuales incurrían considerando que los estudiantes de universidades privadas por ser de carácter público hacen más uso del transporte público no así los estudiantes de universidades privadas que muchos de ellos poseen vehículos propios. Se optó por esta última opción con los resultados siguientes:

Para calcular el gasto anual que hacen los estudiantes tanto de las universidades públicas y privadas se considera los siguientes hechos: 40 semanas de uso de este beneficio en razón que aún sin haber clases los estudiantes se movilizan todo el año. El número de estudiantes universitarios matriculados en universidades públicas es de 107,527 y de las universidades privadas 268,450 (Se ha tomado como referencia la población universitaria del año 2013). Tomando en cuenta la información consignada en las Tablas 5 y 6.

Gasto anual por concepto de pasajes en universidades públicas:

$\begin{array}{lr}0.41 \times 107,527 \times 320= & 14,107,542.00 \\ 0.51 \times 107,527 \times 960 & =52,645,219.00 \\ 0.04 \times 107,527 \times 120= & 516,129.00 \\ 0.04 \times 107,527 \times 40= & 4,301.00 \\ \text { Total } & \text { S/. 67,273,192.00 }\end{array}$

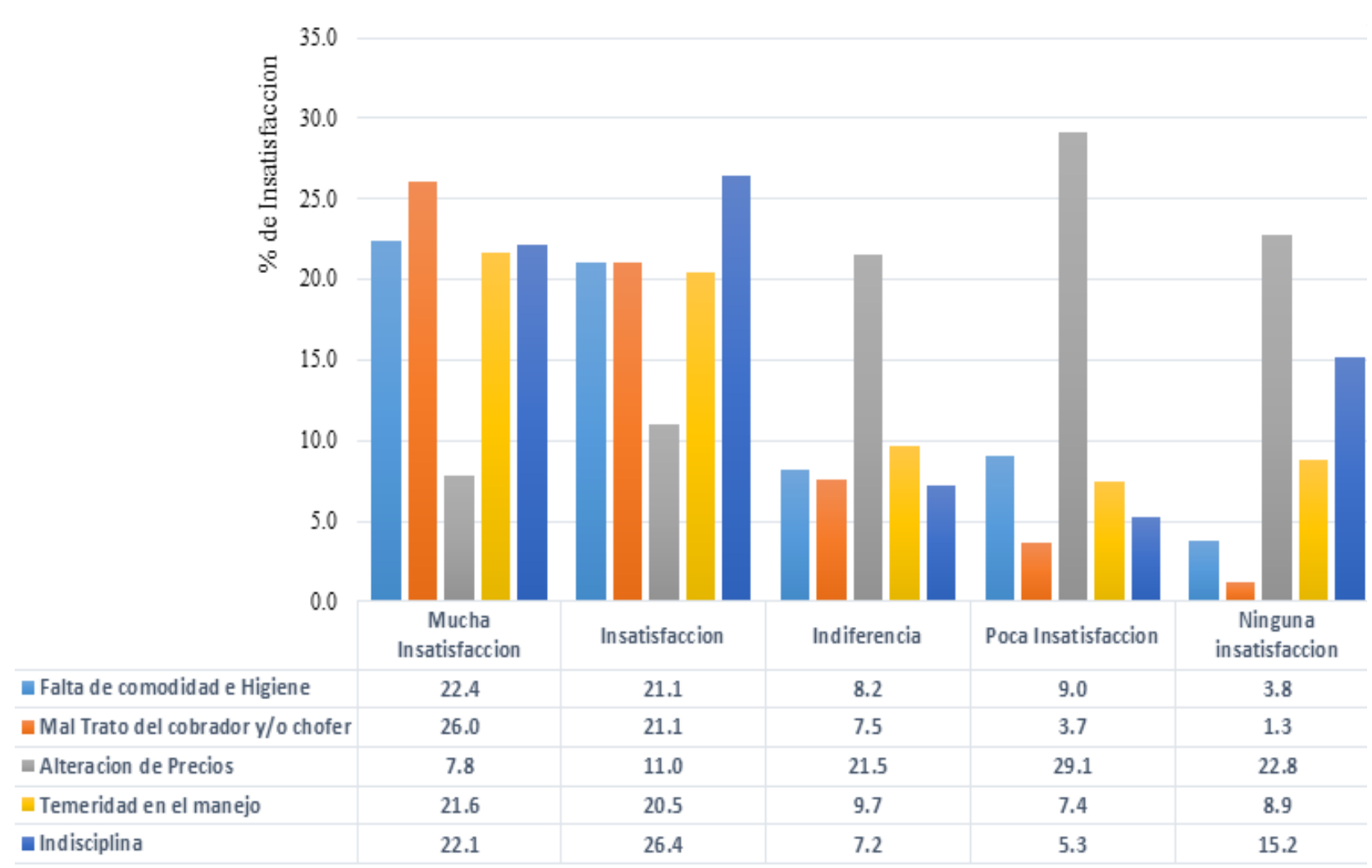

Figura 1. Factores y niveles de insatisfacción de los usuarios universitarios 
Tabla 5. Gasto promedio en pasajes de los estudiantes de universidades públicas

\begin{tabular}{lccc}
\hline Frecuencia de uso & \% Alumnos & Gasto semanal S/. & Gasto anual S/. \\
\hline Una o dos veces al día & 41 & 8 & 320 \\
Más de tres veces al día & 51 & 24 & 960 \\
Menos de 4 veces a la semana & 4 & 3 & 120 \\
Casi Nunca & 4 & 1 & 40 \\
\hline
\end{tabular}

Tabla 6. Gasto promedio en pasajes de los estudiantes de universidades privadas

\begin{tabular}{lccc}
\hline Frecuencia de uso & \% Alumnos & Gasto semanal S/. & Gasto anual S/. \\
\hline Una o dos veces al día & 55 & 8 & 320 \\
Más de tres veces al día & 25 & 24 & 960 \\
Menos de 4 veces a la semana & 12 & 3 & 120 \\
Casi Nunca & 8 & 1 & 40 \\
Elaboración : Propia & & & \\
\hline
\end{tabular}

Participación de las entidades involucradas y de los beneficiarios

Las entidades que regulan el transporte urbano, son entidades públicas que por mandato de la Ley otorgan los permisos correspondientes designando las rutas, márgenes de costos de los pasajes y reglamentos para una circulación ordenada y que beneficie a los usuarios.

Las entidades de transporte urbano, son empresas privadas debidamente constituidas y autorizadas por los organismos reguladores de transporte público (entidades públicas), quienes conjuntamente con el personal que depende de ellas (cobradores y chóferes) dan el servicio de transporte urbano en la ciudad de Lima y Callao.

Los beneficiarios de estos servicios son para este caso los estudiantes universitarios Gasto anual por concepto de pasajes en universidades privadas:

$$
\begin{aligned}
& 0.55 \times 268,450 \times 320=47,247,200.00 \\
& 0.25 \times 268,450 \times 960=64,428,000.00 \\
& 0.12 \times 268,450 \times 120=3,865,680.00 \\
& 0.08 \times 268,450 \times 40=859,040.00
\end{aligned}
$$

Total $116,399,920.00$

Aporte que hacen las empresas públicas y privadas del transporte urbano en la ciudad de Lima al conceder el pasaje universitario

Tomando la información de la Tabla 3 , relacionado con la población estudiantil universitaria del 2013 y los gastos en pasajes expresados por los estudiantes universitarios.Según las tablas 5 y 6 , el aporte que hacen las empresas públicas y privadas del sector transporte urbano al conceder el pasaje universitario es de: $67,273,192.00+116,399,920.00=\mathbf{S} /$. 183, 673,112.00.

Esta cifra corresponde al 50\% del pasaje normal por cuanto es pasaje que pagan los universitarios para movilizarse en forma discrecional a cualquier hora y día.

Es necesario que los usuarios de este beneficio participen en la capacitación de los operadores de los servicios de transporte como una forma de compensación del beneficio recibido y lograr una mejora de la calidad que serán ellos mismo los directamente beneficiados.

Capacitación para el mejoramiento de la calidad del servicio de transporte público urbano en la ciudad de Lima.

El proyecto se formula para comprometer a las universidades en su conjunto cuyo monitoreo inicial estará a cargo de docentes por cada universidad en la que participaran estudiantes de diversos grados y carreras quienes se comprometen a difundir y sensibilizar entre sus compañeros la puesta en marcha del proyecto. quienes por mandato de Ley gozan de un descuento en los pasajes llamado "pasaje universitario" que es en la mayoría de los caso el 50\% de los pasajes para adultos.

El beneficio que gozan los estudiantes universitarios, es otorgado por las empresas de transportes, por mandato de Ley, en cambio los que conforman el sector de empresas de transporte urbano, no reciben ninguna compensación por este tipo de beneficio que otorgan.

La presente propuesta presenta una fórmula que posibilite dar una compensación al sector de transporte urbano de la ciudad de Lima y Callao que permita aprovechar la fortaleza en capacitación que tienen las universidades para ponerlo al servicio de los que están dando el servicio de transporte urbano.

\section{Entidades involucradas:}

\section{Sector público}

Ministerio de Transportes, como órgano regulador del transporte a nivel nacional.

Ministerio del Interior, La Policía Nacional, órgano autorizado para dirigir y sancionar las infracciones de tránsito. Controlan el sistema de transporte bajo directivas emanadas de la autoridad pública.

Ministerio de Educación, entidad encargada de dar la educación en todos los niveles, regula los contenidos de los syllabus.

Universidades Públicas y Privadas, por ser ellas las que tienen la población estudiantil universitaria y participes del beneficio del pasaje universitario.

Municipalidades, órganos que autorizan o conceden las rutas de transporte por sus distritos o provincias, dictaminan las condiciones de la concesión y formulan directivas sobre el uso de las vías públicas dentro de sus distritos

Asamblea Nacional de Rectores o Autoridad equivalente Organismo reconocido como representante y regulador del 
sistema Universitario. Es la única autoridad que entrega y certifica los carnets universitarios

\section{Sector privado}

Empresarios transportistas,personas jurídicas que como personas o empresas son propietarios de los ómnibus o microbuses y combis que prestan el servicio de transporte urbano en la ciudad.

Cobradores, personas encargadas de cobrar los pasajes al interior de los vehículos, tienen trato directo con los pasajeros y son los que informan al conductor donde debe parar y recoger a los pasajeros. Son uno de los principales actores del sistema y el que es menos preparado para la tarea que desarrolla.

Conductores o chóferes, encargados del manejo del vehículo, en algunos casos (muy pocos) son propietarios de los vehículos, en su mayoría son empleados muchos de ellos eventuales que cambian continuamente de patrono o de vehículo por diferentes condiciones ya sea por el trato o la posibilidad de tener un vehículo de mayor capacidad para operar.

\section{Estudiantes universitarios}

Gozan de este descuento por el hecho de ser estudiantes, esta concesión es indefinida mientras conserve su estatus de estudiante, pueden hacer uso las veces que deseen.

Pasajeros o usuarios. Son todas aquellas personas que hacen uso de los servicios de transporte urbano en todo el país.

Entidades financiadoras. Son las entidades que estarían dispuestas a financiar este proyecto.

\section{El ciclo del proyecto y su horizonte de evaluación}

El proyecto se realizara en Lima y Callao por tener la mayor población universitaria $(35 \%)$, se puede monitorear con más facilidad. De obtener resultados satisfactorios del desarrollo de este proyecto, se replicaría en las diferentes ciudades del Perú.

\section{Etapa de Preinversión}

En esta etapa se nombrará el equipo responsable que monitoreará el desarrollo del Proyecto. Este equipo deberá estar integrado por: Dos representantes de las universidades (privada y pública), un representante del Ministerio de Transportes, un representante del Ministerio del Interior, un representante de los empresarios transportistas, un representante de la Municipalidad Metropolitana

Este equipo tendrá como objetivos, elaborar los procedimientos para la puesta en marcha del proyecto y elaborará su propio reglamento de funcionamiento y control.

Se elaborará un censo de las unidades vehiculares así como del personal que presta servicios de transporte urbano en las diferentes empresas inscritas.

Se harán censos en todas las universidades para establecer con el número de estudiantes que tiene cada universidad así como la frecuencia de uso de las unidades de transporte.

Se ubicará los ambientes donde se realizará la capacitación, estableciendo normas que deben de cumplir los ambientes destinados para tal fin.

Se elaborará el perfil que debe tener cada uno de los actores del sistema de transporte urbano (cobradores y chóferes) y establecerá las competencias requeridas para cada uno de estos colaboradores.

\section{Etapa de inversión}

En esta etapa las siguientes actividades que son las que permitirán el desarrollo del proyecto. Adquisición de material didáctico para el desarrollo de la capacitación. Capacitación de los capacitadores, desarrollo del programa de capacitación, evaluaciones periódicas y medición de resultados.

\section{Etapa de Postinversión}

Se deberá fomentar luego de los resultados obtenidos, el proyecto sea auto sostenible y que los mismos actores financien la continuidad del proyecto. En esta etapa se hará una revisión de los resultados para evaluar la posibilidad de replicar proyecto al interior del país.

\section{Demanda de capacitación}

La demanda lo constituyen los trabajadores (chóferes y cobradores) que prestan servicios en las unidades de transporte público urbano. Se estima que en Lima y Callao existen 23,182 unidades, que prestan el servicio de transporte urbano, es decir el mercado de la demanda estaría constituido por 46,364 personas (un chofer y un cobrador por cada unidad de transporte) a lo cual se añadiría 5,000 personas más, que se estima se incorporarían al sistema de capacitación una vez iniciado el programa dada la posibilidad de encontrar una fuente de trabajo digna $\mathrm{y}$ posiblemente mejor remunerada. Lo que haría un total de 51,364 personas que se tendría que capacitar a partir del inicio del programa en forma regular durante los próximos años.

\section{Oferta de los capacitadores}

La oferta está constituida por las universidades localizadas en Lima y Callao.

\section{Balance oferta - demanda}

Las universidades que asumirían este proyecto, son aquellas que están institucionalizadas y como tal sus estudiantes gozan de pasaje universitario. La proporción de chóferes y cobradores que deberá capacitar cada universidad estará en función, a la cantidad de alumnos matriculados que tiene cada universidad. Por cada mil estudiantes que tiene cada universidad deberán de capacitar a los cobradores 
y chóferes de una línea o ruta.Cada línea o ruta tiene un promedio de 60 unidades lo que vendría lo que por cada línea o ruta se debe capacitar a 120 personas. Esta capacitación se hará en dos años para posteriormente hacer actualizaciones permanentes. De acuerdo a esta propuesta el compromiso a capacitar se distribuiría de la siguiente manera:

La Tabla 7 permite establecer las responsabilidades para cada universidad en cuanto a la cantidad de operadores que deben de capacitar así como las empresas concesionarias de las líneas que operan. La propuesta es que cada universidad capacite a los operadores de las líneas que pasan cerca a sus campus o centros de estudio.

\section{Financiamiento}

Por ser un proyecto con intervención pública y privada la participación financiera será con el aporte de las universidades (Tabla 8) en lo referente a las 37 oficinas de capacitación, el mobiliario y equipo para las oficinas es decir en total \$370,000 mientras que la diferencia será financiado por Sistema Nacional de Inversión Pública (SINP) a través del Ministerio de Economía y Finanzas).

\begin{tabular}{ll}
\hline $\begin{array}{c}\text { Entidad } \\
\text { Financiadora }\end{array}$ & Monto \$ \\
\hline SNIP & 647,800 \\
Universidades & 370,000 \\
\hline Total & 917,800 \\
\hline
\end{tabular}

Tabla 7. Distribución para la capacitación de operadores del sistema de transporte urbano Lima

\begin{tabular}{|c|c|c|c|c|}
\hline \multicolumn{3}{|c|}{ No. Alumnos } & \multirow{2}{*}{ Líneas/Rutas } & \multirow{2}{*}{ Pers. a capacitar } \\
\hline \multicolumn{3}{|c|}{ Universidades públicas } & & \\
\hline 1. & Mayor de San Marcos & 29,533 & 30 & 3600 \\
\hline 2. & Ingeniería & 11,376 & 11 & 1320 \\
\hline 3. & Agraria La Molina & 5,055 & 5 & 600 \\
\hline 4. & Federico Villarreal & 23,821 & 24 & 2800 \\
\hline 5. & Enrique Guzmán y Valle & 9,463 & 10 & 1200 \\
\hline 6. & U. Del Callao & 14,005 & 14 & 1680 \\
\hline 7. & José Faustino Sánchez Carrión & 12,827 & 13 & 1560 \\
\hline 8. & Tecnológica de Lima Sur* & 1,448 & 2 & 240 \\
\hline \multicolumn{5}{|c|}{ Universidades privadas } \\
\hline 1. & Pontificia Universidad Católica & 18961 & 19 & 2280 \\
\hline 2. & Peruana Cayetano Heredia & 3865 & 4 & 480 \\
\hline 3. & Del Pacifico & 2382 & 2 & 240 \\
\hline 4. & De Lima & 15421 & 16 & 1920 \\
\hline 5. & San Martin de Porres & 33933 & 34 & 4080 \\
\hline 6. & Femenina Sagrado Corazón & 2573 & 3 & 360 \\
\hline 7. & Inca Garcilaso de la Vega & 29133 & 30 & 3600 \\
\hline 8. & Ricardo Palma & 16562 & 17 & 2040 \\
\hline 9. & Peruana Unión & 5909 & 6 & 720 \\
\hline 10. & Marcelino Champagna & 1013 & 1 & 120 \\
\hline 11. & Peruana de Ciencias Aplicadas & 16946 & 17 & 2040 \\
\hline 12. & San Ignacio de Loyola & 8445 & 9 & 1080 \\
\hline 13. & Alas Peruanas & 62974 & 63 & 7360 \\
\hline 14. & Norbert Wiener & 5413 & 6 & 720 \\
\hline 15. & San Juan Bautista & 9199 & 9 & 1080 \\
\hline 16. & Tecnológica del Perú & 13949 & 14 & 1680 \\
\hline 17. & Científica del Sur & 3115 & 3 & 360 \\
\hline 18. & Católica Sedes Sapientiae & 4641 & 5 & 600 \\
\hline 19. & ESAN & 1544 & 2 & 240 \\
\hline 20. & Peruana de las Américas & 2188 & 2 & 240 \\
\hline 21. & Peruana de Ciencias e Informática & 1030 & 1 & 120 \\
\hline 22. & Antonio Ruiz de Montoya & 346 & 1 & 120 \\
\hline 23. & Telesup SAC & 1826 & 2 & 240 \\
\hline 24. & Sergio Bernales SAC * & 579 & 1 & 120 \\
\hline 25. & Peruana Simón Bolívar* & 54 & 1 & 120 \\
\hline 26. & De Ciencias y Humanidades $*$ & 1492 & 1 & 120 \\
\hline 27. & Peruana de Integración Global * & 826 & 1 & 120 \\
\hline 28. & Autónoma del Perú* & 1798 & 2 & 240 \\
\hline 29. & Jaime Bauzate y Meza * & 1627 & 2 & 240 \\
\hline
\end{tabular}

Temas y evaluación sobre la capacitación

Los temas a desarrollarse así como la frecuencia y detalles de los cursos o materias serán previamente diseñados en coordinación con el Ministerio de Educación y los programas de proyección social de cada universidad las evaluaciones corresponderán realizarlas a la Unidad Ejecutora que será nombrada por el Gobierno para poner en práctica el proyecto, quienes darán cuenta a sus mandantes sobre los resultados de estas evaluaciones.

\section{Evaluación a nivel social}

a) Cuál es la percepción de la sociedad sobre los resultados del proyecto.

b) Si se ha logrado integrar en esta área al sistema de transporte con las Universidades

c) $\mathrm{Si}$ es factible replicar esta experiencia en provincias.

d) $\mathrm{Si}$ es posible replicar esta experiencia en otras aéreas. Ejemplo la práctica de valores, la puntualidad, la diligencia etc.

Frecuencia:

Se efectuará una evaluación por año.

\section{Inicio del proyecto}

Universidad Nacional Agraria La Molina (UNALM) como universidad podría convertirse en 
protagonista del desarrollo de este proyecto considerando que tiene (año 2013) en total 6426 estudiantes (5261 en pregrado y 1165 en postgrado)

De otro lado existen más de 11 líneas que pasan por la puerta de la universidad lo que hace que sirva como referencia para los transportistas y hasta cierto punto una parcial identificación con las UNALM.

Tomando en consideración la Tabla 9 donde se propone asignar un número de rutas para capacitar de acuerdo a la población estudiantil de cada universidad, a la UNALM le correspondería capacitar a 5 líneas o rutas (cada línea o ruta tiene 60 unidades de transporte y cada unidad de transporte tiene dos operadores) es decir a 600 personas.

Tabla 8. Presupuesto aproximado por el funcionamiento del proyecto para la capacitación del personal dedicado al transporte urbano en Lima

\begin{tabular}{|c|c|c|c|}
\hline Cantidad & Concepto & Mensual \$ & Anual \$ \\
\hline 1 & $\begin{array}{l}\text { Alquiler de oficina para el } \\
\text { funcionamiento de la comisión }\end{array}$ & 1,000 & 12,000 \\
\hline 37 & $\begin{array}{l}\text { Oficinas para la capacitación, } \\
\text { una por cada universidad } \\
(\$ 500.00) \mathrm{c} / \mathrm{u}\end{array}$ & 18,000 & 216,500 \\
\hline 1 & $\begin{array}{l}\text { Muebles y equipos de oficina, } \\
\text { para la oficina de Monitoreo }\end{array}$ & & 2,000 \\
\hline 37 & $\begin{array}{l}\text { Muebles y equipo de oficina, } \\
\text { para los centros de capacitación } \\
(\$ 2,000.00) \mathrm{c} / \mathrm{u}\end{array}$ & & 74,000 \\
\hline 1 & $\begin{array}{l}\text { Gerente general, oficina de } \\
\text { Monitoreo }\end{array}$ & 5,000 & 60,000 \\
\hline 5 & $\begin{array}{l}\text { Dieta para los directores, oficina } \\
\text { de monitoreo }(\$ 300.00) \mathrm{c} / \mathrm{u}\end{array}$ & 1,500 & 18,000 \\
\hline 144 & $\begin{array}{l}\text { Capacitadores ( } 4 \text { por cada centro } \\
\text { de capacitación) (\$300.00) c/u }\end{array}$ & 44,400 & 532,800 \\
\hline 10 & $\begin{array}{l}\text { Personal que levantara la } \\
\text { información relacionada a } \\
\text { verificación de datos estadísticos } \\
\text { durante } 30 \text { días }\end{array}$ & 3,300 & 3,300 \\
\hline & Total & & 917,800 \\
\hline
\end{tabular}

\section{Empresas con las que se puede empezar}

Se ha hecho una investigación sobre las empresas que son más usadas por los estudiantes de la UNALM, y se ha encontrado que las preferidas por los estudiantes, son aquellas que pasan por la puerta principal, y son las siguientes:

Cada una de estas empresas tiene un promedio de 60 unidades operativas y dan entre 3 a 4 vueltas al día, en cada vuelta emplean entre 2 y dos horas y media con un recorrido aproximado por cada unidad entre 400 a 500 Km, Según las investigaciones realizadas, el chófer tiene un ingreso entre 150.00 y 200.00 nuevos soles diarios dependiendo del número de vueltas que haya realizado en el día, de igual manera el cobrador tiene un ingreso entre $80.00 \mathrm{y}$ 120.00 al día (siempre y cuando rinda cuenta documentada al propietario), normalmente esta cifra es mayor por lo que el cobrador trabaja un día para descansar dos.

La unidad de proyección social juntamente con la
Federación de Estudiantes de la UNALM podría empezar a sensibilizar entre los actores internos y externos para poder dar inicio a este proyecto.

Tabla 9. Empresas de transporte urbano con quienes se puede iniciar el proyecto

\begin{tabular}{|c|c|c|}
\hline $\begin{array}{c}\text { Nombre de la } \\
\text { Empresa }\end{array}$ & Ruta Asignada & Recorrido \\
\hline Empresa & Línea EM-48 A & $\begin{array}{c}\text { San Juan de Lurigancho- } \\
\text { Pachacamac }\end{array}$ \\
\hline $\begin{array}{l}\text { Negociaciones } \\
\text { Santa Anita S.A }\end{array}$ & Línea EM-48 & $\begin{array}{c}\text { San Juan de Lurigancho- } \\
\text { Pachacamac }\end{array}$ \\
\hline $\begin{array}{l}\text { Empresa } \\
\text { Turismo Cinco } \\
\text { Estrellas S.A }\end{array}$ & Línea $48 \mathrm{~B}$ & $\begin{array}{c}\text { San Juan de Lurigancho- } \\
\text { Pachacamac }\end{array}$ \\
\hline $\begin{array}{l}\text { Empresa Ettsa } \\
\text { Red Lima Movil }\end{array}$ & Línea EM-58 A & Pachacamac-La Victoria \\
\hline $\begin{array}{l}\text { Empresa } \\
\text { Ettsromsa }\end{array}$ & Línea EM-58 B & Pachacamac-La Victoria \\
\hline $\begin{array}{l}\text { Empresa } \\
\text { Etramusa }\end{array}$ & Línea IO-72 & Musa-Callao \\
\hline $\begin{array}{l}\text { Empresa Metro } \\
\text { Vias }\end{array}$ & Línea OM-30 & Cieneguilla-Callao \\
\hline $\begin{array}{l}\text { Empresa Los } \\
\text { Magnificos }\end{array}$ & Línea EQ-69 & Pachacamac- La Victoria \\
\hline $\begin{array}{l}\text { Empresa } \\
\text { Etysermulma S.A }\end{array}$ & Línea EO-69 & $\begin{array}{c}\text { San Juan de Lurigancho- } \\
\text { Pachacamac }\end{array}$ \\
\hline $\begin{array}{l}\text { Empresa Et } \\
\text { Molinero } \\
\text { Express S.A }\end{array}$ & Línea IM-49 & Cieneguilla-Callao \\
\hline $\begin{array}{l}\text { Empresa } \\
\text { Etchusac }\end{array}$ & Línea IO-71 & Ventanilla-La Molina \\
\hline
\end{tabular}

\section{Conclusiones}

El público en general y los estudiantes universitarios se encuentran insatisfechos con el servicio de transporte urbano, por el pésimo servicio que brindan. A pesar de ello, la población de estudiantes universitarios en la ciudad de Lima ha tenido un incremento del 15\% (325,052 en el 2008 a 375,977 en el 2013). Esta población se irá incrementando en los próximos años.

El gasto anual por concepto en pasajes que realizan los estudiantes universitarios de universidades públicas y privadas (con una aproximación muy conservadora) en la ciudad de Lima es de S/ 183.6 millones de soles que corresponde al pago del $50 \%$ del pasaje universitario.

El monto anteriormente señalado corresponde prácticamente a una subvención que hacen las empresas privadas de transporte público, al sistema universitario (estudiantes) pero, estas empresas no reciben nada a cambio de esta concesión.

No hay registros de hechos ni intentos concretos que se hayan realizado para mejorar la calidad del transporte urbano, sino por el contrario por razones de demanda se ha incrementado la proliferación de empresas y junto con ello la calidad ha disminuido.

La UNALM está en condiciones de iniciar este proyecto con la experiencia ganada en los cursos de extensión a la sociedad 


\section{Literatura citada}

Gerencia de Transporte Urbano de la Municipalidad Metropolitana de Lima. 2013. Recuperado de: http:// www.munlima.gob.pe/gerencias/item/27710-gerenciade-transporte-urbano

Instituto Nacional de Estadística e Informática. 2016. Número de alumnos matriculados en universidades. Recuperado de: https://www.inei.gob.pe/estadisticas/ indice-tematico/university-tuition/ 